\title{
IoT based Big data Analytics in Healthcare: A Survey
}

\author{
Aiswarya $\mathrm{S}^{1}$, RameshK ${ }^{2}$, Sasikumar $\mathrm{S}^{3}$ \\ Research Scholar, Department of Computer Science, HITS \\ Email: 1 aiswarya.sudha@outlook.com \\ Professor, Department of Computer Science, HITS \\ Email: ${ }^{2}$ Ramesh.me@gmail.com \\ Professor, Department of Electronics and Communication, HITS \\ Email: ${ }^{3}$ ssaik@hindustanuniv.ac.in
}

\begin{abstract}
Heterogeneous data surround the world, and it is generated enormously in every moment of a day from multiple sources. These enormous amounts of unstructured, structured, and semi-structured data are called big data, and this cannot store in conventional database systems. Big data, IoT plays a significant role, here big data implies the clinical data which comes in the form of medical prescriptions, laboratory data, genome database, electronic health records, medical images, medical IoT, etc. It supports clinical decisions, along with advanced and personalized health care. In this paper, a brief survey is from the related projects which deal with the challenges, techniques, as well as the different directions in big data health care.
\end{abstract}

Keywords: Healthcare, Big data Analytics, IoT, Fog Computing

\section{Introduction}

Big data is not just a term, and it is a complete subject with tools, techniques, and frameworks. It's a vast amount of data that keeps on growing with time. It comprises numerous other methods such as data storage, data mining, data analysis, sharing data, and visualization. Big data offers a way to find a new perception of the existing data and directions to catch and analyse succeeding data.

Big data has been used in different fields by the researchers to pillar their findings and conclusions. Organizations can enhance customer expectation by gathering big data and produce products which meet their desires. Through resource optimization, they can improve their operational efficiency and productivity [1]. The transport industry, using technologies of Big data for enhancing quality services, traveller gratification, and management [2]. Also, it can advise customer feedback service. In the education field, it plays a role in influencing students' behaviour and engagement also educational institutions can work big data along with predictive analytics for getting the future insights of the student's outcome [3].It used for the effective monitoring of smart grid operations and airline route optimization [4-6]. The Agricultural field is covering various applications of IoT, big data [7-8]. Big data made an evolutionary change in the healthcare industry by using its techniques and technologies in different formats of data sets, including structured, semi-structured, and unstructured. These medical data are in digitized formats and produce rapidly [9]. Several surveys say that the medical data in the zettabyte scale and soon it touches the yottabyte. In the health domain, Big data reduces the treatment cost and provides a better dimension to the diagnostics and choice making. The main attractions of Big data are its fantastic number of data sources and diversity [10-11]. If we categorize it, we will get different groups. Today's data is extracted from five primary sources: IoT, Mobile devices, Enterprise information systems, Social networks, and public and open data. There are approximately billions of smartphones used widely around the world, and these portable devices are significant donors to Big data. Similarly, the IoT devices, like sensors, connected with humans, generate a large data set.

In this paper, the first section discussed the challenges facing in the health domain, the further article organized as Related works, and Basic concepts. The final section of this study has identified various IoT big data Applications in Healthcare and continued by the conclusion.

\section{Challenges in health care}

The volume of Medical data is increasing day by day, and it's unlike other data [12]. They are difficult to access quickly and sensitive data because of their rapid generation. The quality of trustworthiness, consistency, and security of medical data is the most significant challenges they are facing [13-16]. Here arises the need for realtime streaming technologies and tools [8][17]. Predictability and on-time processing have a significant role in this field [16]. The integrity and interoperability of health data are other areas that need attention [13][18][19][20]. Now a day's health sectors want intelligence in their field [21]. Accuracy needs in equipment used for disease detection. Systems using in this field need better memory capacity, latency, bandwidth [22]. Audio pathology can be monitor by the help of a Cloud IoT integration system and using this technology; 
extensive data can manage [23]. Patients information should be private and secure [13]. Recent researches say that using the social network system for health care adds more benefits, and it is a challenging and exciting job to extract and collaborate these data for health wellness. One of the probable challenges is intricate a health verdict system for predicting and estimating the epidemic dispersion around the world by social media mining. For making this predictable system possible, we need to consider related attributes to the epidemic or pandemic like corona, SARS, influenza, etc. The values extracted in the form of comments, posts, likes, sharing. These past values can make a predictive system that can use it later [24]. The enormous power of Vast data, along with the wide variety of social media data, can merge and provide an effective predictive system

\section{Related works and basic concepts}

\subsection{Big Data: Process and Characteristics}

In recent years, a vast amount of data produced around the world and eighty percentage of such data are unstructured in the format of video, audio, images, text, and these types of heterogeneous data referred to as Bigdata. These data can be grouped into transactions, machine-to-machine, biometric, social media/web, and human-generated data. The process mainly includes Big data Generation, Acquisition, which provides for data collection, transmission and pre-processing, Storage, and Analysis. The attributes which define big data are called V's of big data. There are $4 \mathrm{~V}$ 's plays a major role, and they are the following:

- Volume: It is the gigantic data that can be range from Tera Bytes to Zetta Bytes or even more generated in every minute from multiple resources [25].

- Variety: It refers to the heterogeneous data. Data can be represented in different ways, such as structured, semi-structured, and unstructured data, which can include image, text, audio, and video.

- Velocity: It describes the speediness of data generation, collection, and examination. Today's data is time-sensitive and needs to handle at the time. It analyses the streaming data in real-time [9].

- Veracity: It describes the reliability and accuracy of the data. Collecting tons of data are of no use if it is not trustworthy. It is the major challenge comparing with volume and velocity.

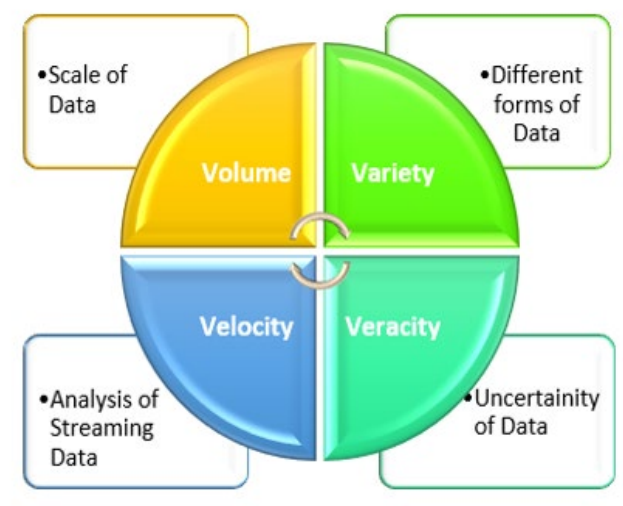

Fig. 1. Four V's of Big Data (Source: [30])

Figure 1 shows the significant four V's of Big data.

\subsection{Big Data Analytics}

Big data analytics retrieve relevant information from the ocean of data. It analyses the various types of data sets and retrieves useful information. Big data analytics mainly used or prediction and forecasting [26-27]. It also finds the hidden patterns, consumer choices, and market trends for better decision making in organizations. Data mining is the central concept using analytics. There are different technologies used in big data analytics. Among them, MapReduce plays a significant role in various fields like online streaming data analytics, Machine to Machine, IoT data analytics, etc. In healthcare analytics, different algorithms like Machine learning, deep and cognitive learning using with the help of artificial intelligent and artificial neural networks [24]. It includes statistical and mathematical approaches for understanding and forecasting the results. There is much opensource software that serves the purposes of analysing processing. Among them, the Apache software foundation 
is most popular, and it provides a set of open-source frameworks that support the data storing and analysis called Hadoop [24].

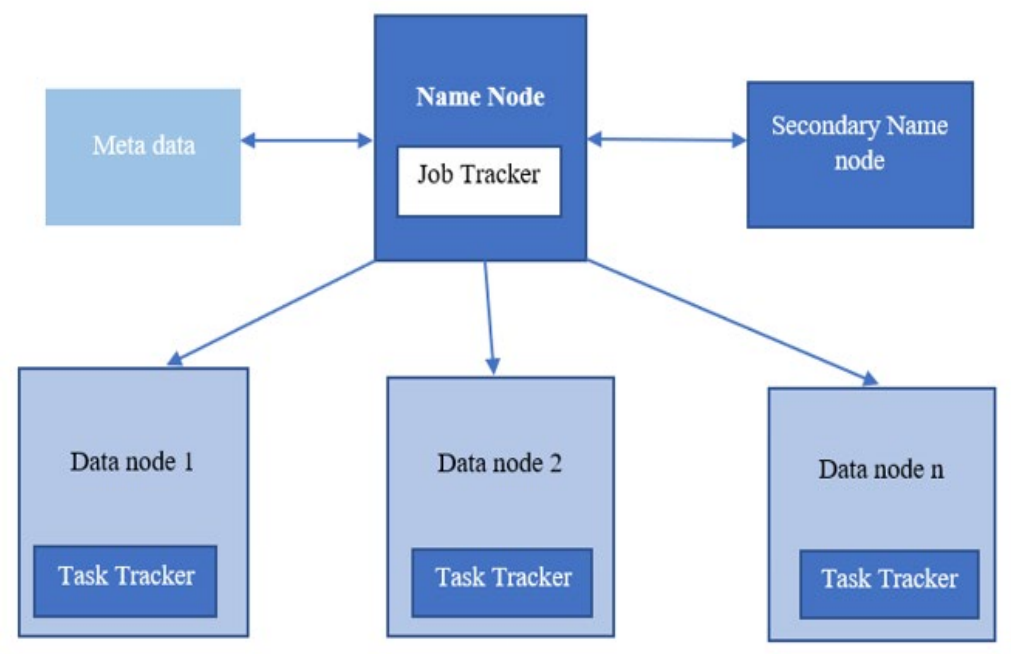

Fig. 2. Hadoop File System Daemons (Source: [40])

Figure 2 shows the Master-Slave architecture concept of Hadoop, which is having only one Name node and many Data nodes.

- Name Node: Act as a master and assign jobs across the nodes. It has the metadata which defines the other data.

- Secondary Name Node: It is the checkpoints metadata on the name node, and name namespace of name node. It is not a backup for the name node.

- Data Node: Act as a slave and executes all the tasks assigned by the Name node, which is the master.

- Job Tracker: Assigns the jobs to slave nodes get acknowledged.

- Task Tracker: Performs the jobs given by the job tracker and monitor the completion.

Storing is one of the significant issues facing bigdata, and some of the popular technologies used to store and analyse the data shown in Table 1 below.

Table 1. Comparison of Big data Technologies (Source: BI reporting, Scott Michell)

\begin{tabular}{|c|c|c|c|c|}
\hline Features & HBase & Cassandra & MongoDB & Hive \\
\hline Year implemented & 2008 & 2008 & 2009 & 2012 \\
\hline Open-source & Yes & Yes & Yes & Yes \\
\hline $\begin{array}{l}\text { Implemented } \\
\text { Language }\end{array}$ & Java & Java & $\mathrm{C}++$ & Java \\
\hline Developer & $\begin{array}{ll}\text { Apache } & \text { Software } \\
\text { Foundation } & \end{array}$ & $\begin{array}{ll}\text { Apache } & \text { Software } \\
\text { Foundation } & \end{array}$ & MongoDB, inc & $\begin{array}{ll}\text { Apache } & \text { Software } \\
\text { Foundation } & \end{array}$ \\
\hline Definition & $\begin{array}{l}\text { The non-relational } \\
\text { distributed database } \\
\text { based on Apache } \\
\text { Hadoop and Bigtable }\end{array}$ & $\begin{array}{l}\text { The non-relational } \\
\text { distributed database } \\
\text { based on DynamoDB } \\
\text { and Bigtable }\end{array}$ & $\begin{array}{l}\text { Most popular NoSQL } \\
\text { database, Document } \\
\text { store }\end{array}$ & $\begin{array}{l}\text { Data Warehouse for } \\
\text { distributed data sets } \\
\text { on Hadoop, which } \\
\text { uses relational DB. }\end{array}$ \\
\hline Operating System & $\begin{array}{l}\text { Windows, Linux, } \\
\text { Unix }\end{array}$ & BSD & $\begin{array}{ll}\text { Windows, } & \text { Linux, } \\
\text { Solaris, OSX } & \\
\end{array}$ & $\begin{array}{l}\text { All OS, which } \\
\text { supports java VM. }\end{array}$ \\
\hline Schema free & Yes & Yes & Yes & No \\
\hline SQL & Not & Not & Not & Not \\
\hline Typing & No & Yes & Yes & Yes \\
\hline Secondary Indexes & No & Restricted & Yes & Yes \\
\hline Durability & Durable & Durable & Durable & Durable \\
\hline MapReduce & Yes & Yes & Yes & Yes \\
\hline $\begin{array}{l}\text { Access methods/ } \\
\text { APIs }\end{array}$ & $\begin{array}{l}\text { Java API, HTTP API, } \\
\text { RESTful }\end{array}$ & Proprietary protocol & $\begin{array}{l}\text { JSON } \quad \text { using } \\
\text { proprietary protocols }\end{array}$ & JDBC, ODBC, thrift \\
\hline Partition method & Shard & Shard & Shard & Shard \\
\hline Server-side scripts & Yes & No & Java script & Yes \\
\hline
\end{tabular}




\begin{tabular}{|l|l|l|l|l|}
\hline User rights & Access Control List & $\begin{array}{l}\text { User can define per } \\
\text { object }\end{array}$ & $\begin{array}{l}\text { User Can define full } \\
\text { access }\end{array}$ & $\begin{array}{l}\text { Rights for users, } \\
\text { groups, roles }\end{array}$ \\
\hline
\end{tabular}

\subsection{Fog Computing}

A new area Internet of things leads to big data analytics to various computing like parallel, distributed, cluster, cloud, and Fog computing. The concept of Fog computing arisen in 2005. In 2014, cisco introduced this word as a continuation of the cloud. The large and high-speed data production from IoT devices cannot efficiently be handled by the central storage hubs. Cloud computing came up with an answer to this problem, but again bottlenecks such as security threats and low latency issues when it comes toa medical emergency, transaction verifications, etc. Fog acts as a cache memory between the user and the cloud. It provides the quality of services to real-time applications that need low latency [24][28]. The key distinguishing factor of fog computing from cloud computing is its distributed decentralized system [25]. The applications which needed predictable and low latency are highly beneficial from fog computing. From 2017 onwards, the scope of fog computing in the research field started blooming and various new topics added to it, like wireless sensors, wearable devices, body area networks, interoperability, and energy-efficient models [29-30]. Fog layer stores only the essential information and sends the exact information to the cloud for big data analysis and storage. A device can act as fog node if it is having storage, computing, and connectivity facilities [28] [31-32]. There are six layers present in fog computing: Transport layer, Security layer, Temporary layer, Pre-processing, monitoring, Physical, and virtualization layer.

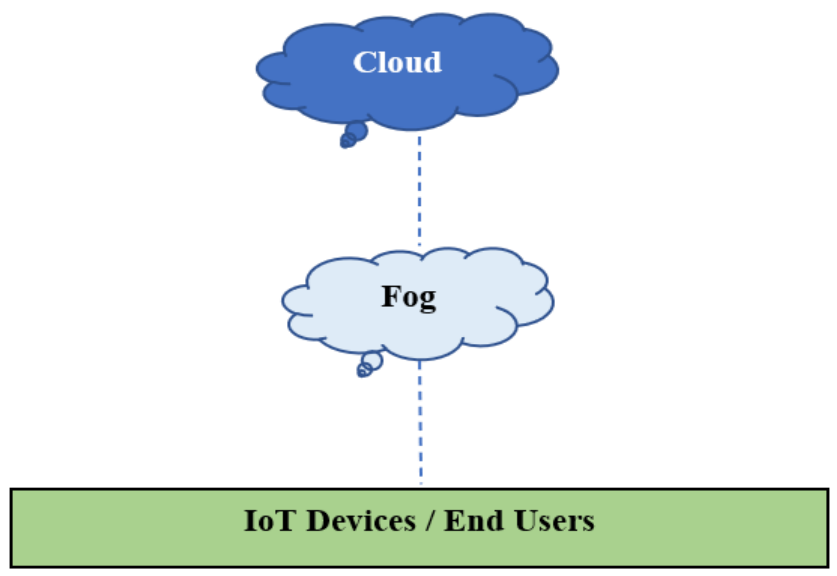

Fig. 3. Fog Computing view (Source: [38])

Figure 3 shows how the fog act as a link between cloud and IoT devices.

\subsection{Internet of Things [IoT]}

Today the world is linked with the Internet. With the arrival of the concept Internet of things, everything related to anything. IoT widely spread over the globe and connected through the Internet. Our health care industry highly benefited from these intelligent devices [33]. It provides a predictive and intelligent system which tracks people and object [23]. Identify and authenticate people and automatically collect data through sensors. It reduces the cost of healthcare and provides a sound care system. Merging of IoT Big data model brings a drastic change in the health care industry. It enables smart healthcare management. 


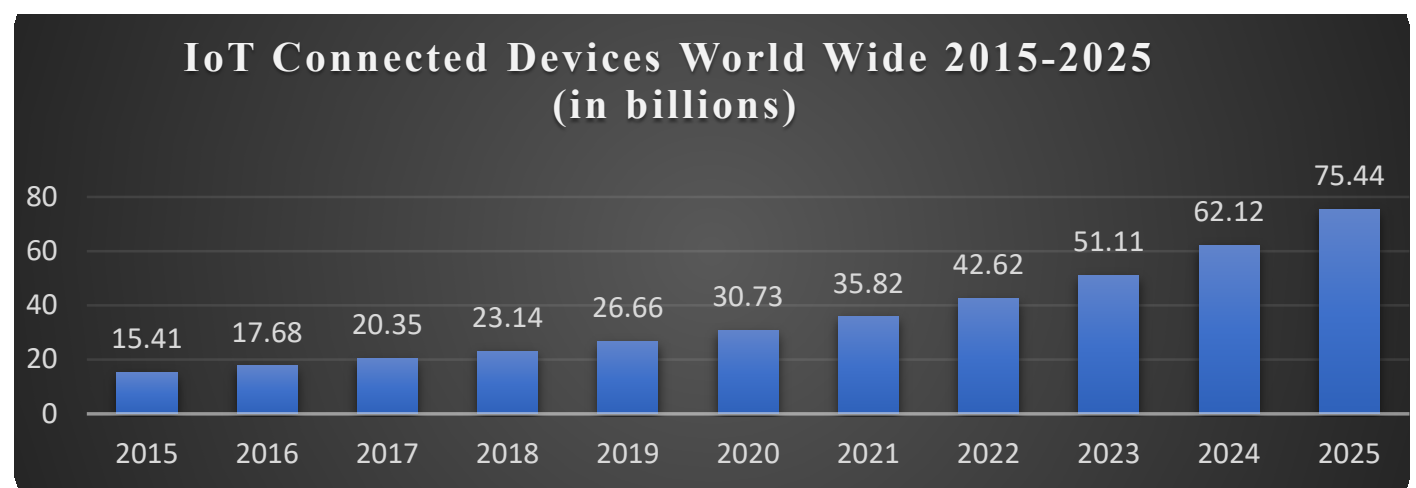

Fig. 4. IoT connected devices across worldwide from 2015- 2025 (Source: [15])

Figure 4 [15] shows from 2015 onwards, the concept of IoT Big data convergence concept gets start popularising.

By adopting this technology, patients get early detection of diseases, regular monitoring, and better services [28] [34-35]. IoT enables remote monitoring with an alert system, which is highly beneficial for caring elders and physically challenged ones [36]. The IoT components can discuss in to mainly three: IoT hardware, middleware, and presentation [29]. IoT software and protocols are the other two essential components.

\subsubsection{IoT Hardware}

It is difficult to show a well-defined hardware structure or architecture of IoT. It will change according to the needs. But we have the basic building blocks for the hardware, and they are Thing, Data acquisition, processing, and Communication unit [37]. Figure 5 shows the basic building blocks for the IoT hardware.

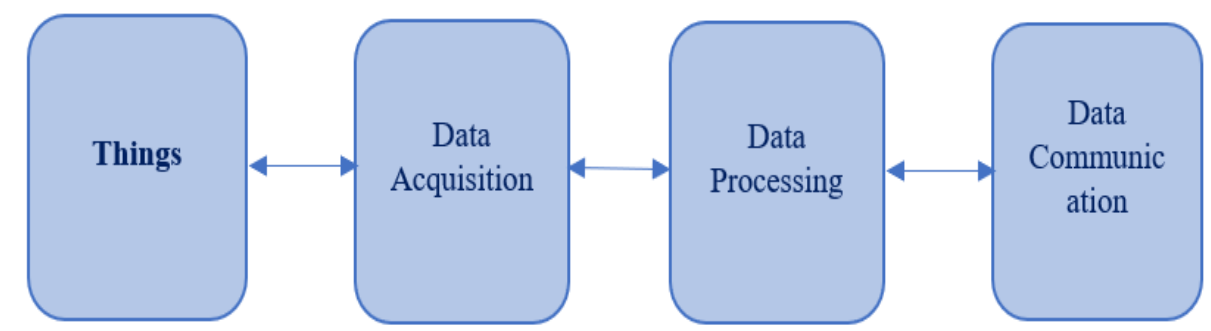

Fig.5.Basic units of IoT Hardware (Source: [37])

Thing: It is what you want to regulate or display. It integrated with devices always. These smart devices further connected with the cloud [38].

Data acquisition: This module receives physical signals from the thing and converts to digital signals that can be changed by the system, in this unit only all the sensors acquiring the signs such as temperature, light, motion, vibration, etc.

Data Processing: The computers process the data and do the analytical parts and stores locally. There are two things we need to focus on in this module, i.e., Processing power and the size of the hardware [39]. How much local storage do we need?

Data Communication: This module enables interaction with the cloud platform. It includes communication ports like USB, CAN, serial, and also wireless technologies like Wi-Fi, ZigBee, etc. Gateway architecture also used for communication.

\subsubsection{IoT Middleware}

It permits the connectivity of sensors with application layers. Data Communicate over networks. IoT middleware needs to support large and dynamic numbers of endpoints, streaming data, a considerable volume of simultaneous messages, new protocols, real-time processing, and execution models [19]. 


\subsubsection{IoT Presentation}

It rests on the data lifecycle, starts from the production of data to analysis of data. It is significant to present data to act on it. Stable and secure communication and a sharable representation of data are the main challenges met in interaction over IoT networks. There are two main characteristics of representation [40]: understandability, and their visualization and interpretability.

\subsubsection{IoT Software and Protocols}

Through embedded systems, middleware, and platforms, IoT software provides actions and networking. It enables the real-time analysis of huge data that is producing from IoT devices. It collects data through various mediums such as real-time sensors, protocols, M2M networks to provide different kinds of services application extension used for producing more accurate and reliable data [41]. All the components of IoT include a mobile, router, and cloud systems linked by a group of IoT protocols. It enables interaction between the systems.

Table 2 shows the eight layers of the critical IoT protocols, which are developed by the Institute of Electrical and Electronics Engineers (IEEE)and the European Telecommunications Standards Institute (ETSI).

Table 2. IoT layers with Protocols

\begin{tabular}{|l|l|l|}
\hline No & \multicolumn{1}{|c|}{ Layers } & \multicolumn{1}{c|}{ Protocols } \\
\hline 1 & Identification & IPv6, EPC, URIs, uCode \\
\hline 2 & Infrastructure & IPv4/IPv6, 6LowPan, RPL \\
\hline 3 & Transport & Bluetooth, Wi-Fi, LPWAN \\
\hline 4 & Discovery & DNS-SD, Web, Mdns \\
\hline 5 & Data Protocols & MQTT,Node, WebSocket, CoAP \\
\hline 6 & Device Management & OMA-DM, TR-069 \\
\hline 7 & Semantic & WebThing Model, JSON-LD \\
\hline 8 & Multi-layer Frameworks & Weave, IoTivity, Homekit, Alljoyn \\
\hline
\end{tabular}

\section{Applications of health care IoT Big data}

The smart healthcare concept begins with the convergence of the IoT Big data model. A wide variety of applications are going to discuss in this part of the paper.

\subsection{Internet of Medical Things (IoMT).}

A new branch emerges for smart healthcare-associated tracking and research called The Internet of medical things (IoMT). It is a heterogeneous wireless communicating system for digitized healthcare schemes, which links the medical resources and services. IoMT stores and tracks vital medical and statistical information. Health data ranges from low to high speed and diversity such as Doctors/ medicinal prescriptions, lab data; Machine produced data, admin data, and insurance data [42]. The components of IoMT are biosensors, communication gateways, and server/ cloud [14][38]. The main advantage is that improved quality and cost-effective health care of patients, which supports a shift from hospital to home.

\subsection{Remote and Real-Time Health Monitoring}

The main attractive feature of big data IoT in the health domain is remote and real-time analysis of individuals' fitness status. Real-time epidemic and pandemic can be monitor and forecast effectively and track people's locations easily using these technologies. These data were processed and filtered regularly, then upload and forecast in the cloud publicly where most of the health monitoring systems built [43]. The vulnerable parts like infants, pregnant women, elderly persons [44], or chronic patients need more focus [45]. It achieved by the collaboration of IoT, Fog, and cloud services and can send emergency signals if required [46]. Sensors capture the real-time data and send it either to Fog or to cloud according to the nature of data. Health checking devices can range from blood pressure monitor [47-48][18]to an advanced pacemaker monitor. Fitbit wristbands and innovative hearing aids are now part of people's day today life.

\subsection{Prevention and diagnosis}

As we know, "Prevention is better than cure," our goal is to find a healthy way that needs the timely management of health issues, which is essential. For chronic cases, early detection and diagnosis help a lot. 
Delivering accurate data at the time of treatment creates a harmless, relaxed, and profitable recovery for patients. Point of care systems enabled with IoT are highly beneficial for timely and reliable diagnosis of patients [49]. One of the studies which are part of the project EDUCERF [50][53] uses smart toys for finding early detection of difficulties in children. Smart toys, along with the sensors they can early monitor and detect. Similar studies also have done by authors [51][54] proposed a work called Mining minds for custom-made support for the prevention of illnesses. It comprises big data, IoT wearable sensors, cloud computing, and knowledge analytics.

\subsection{Medical emergency alert system}

The unavoidable requirement for health emergencies is rapid actions. Real-time health emergency systems integrated with the IoT sensors. Wireless Body-Based Area Network (WBAN) is connecting with patients [9]. This emergency alert system is more helpful to the elderly group. Because these intelligent systems allow regular monitoring, wireless sensor networks (WSN), immense data, and cloud computing, it generates an emergency call whenever it detects the unusual activities like falling, Wheezing, etc [46].

\subsection{Inference systems in Ubiquitous sensing}

There are always challenges like the battery life of mHealth devices in ubiquitous sensing of the patient's status by smart devices. In 2017, an interface system developed [52] part of their study, which effectively and accurately collect and transfer the body sensor data to IoT networks and mHealth. Also, during the quarantine period, it is effectively monitoring the symptoms [53-54]. The sensor-embedded interference system transfers the optimized data to avoid data congestion in the network when it is in the case of a geographical area or a massive number of people.

\subsection{Better Healthcare Administration}

With the help of Big data, Hospital administration runs much more comfortable, and it helps to reduce the costs. It can provide the best healthcare support and risk management. Medical experts can analyse the data and conclude effective treatments. There are bigdata used intelligent applications that help to boost their business. Big data can prevent errors like wrong medicine, usage, and human errors made by the administrators. Also, it can find fraudulent.

\section{Conclusion}

This paper shows how the Internet of Things and Big data technologies and the deeply intertwined with each other for health wellbeing. This study provides recent trends in research over the Bigdata IoT healthcare domain including the job of Bigdata and IoT, Comparison of technologies and tools used for it, Different layers of fog computing over the cloud, IoT infrastructure with layers and protocols, Applications and issues of big data IoT in the health domain. From this study, it shows that the health domain is the area where a single minute is a matter, needs rapid actions for life-saving. So, storage of the analytics data from the different IoT bigdata devices over the cloud needs much attention, and it is one of the main challenges our technology facing. As a solution from our findings, Fog can act as an intermediate layer that handles only the critical data, and the remaining parts of data sent to the centralized cloud. Fog can store and process quickly upon its application needs. Health sensitive data that needs rapid action can depend on this fog layer because of its low latency. The study of Big data IoT can make a drastic change in our society by its tremendous capabilities, which is yet to explore. It's an opportunity for the researchers as well as health workers to do future enhancement. Finally, it is concluding from the survey that, apart from the existing ones, it needs to find more efficient, secure, and reliable paradigms of Big data IoT for the betterment and wellbeing of the Human race.

\section{References}

[1] N Khan et.al. Big Data: Survey, Technologies, Opportunities, and Challenges, The Scientific World Journal, 2014, pp. 01-18.

[2] W-K. Liu et.al. Optimizing bus passenger complaints service through big data analysis for improved public sector management MDPI Journal, 2016, Vol 8 (Issue 12), pp. 01-21.

[3] R.J. Watson et.al. Big Data and Student engagement among vulnerable youth: A review, Current Opinion in Behavioural Sciences,2017, vol 18, pp. 23-27. 
[4] H Daki et.al. Big Data Management in smart grid: Concepts, Requirements and Implementation, Journal of Big Data,2017, vol 4 (Issue 13), pp. 01-19.

[5] E. Kasturi et.al. Airline Route Profitability analysis and Optimization using BIG DATA analytics on aviation data sets under heuristic techniques, Fourth International conference on recent trends in Computer science and Engineering (ICRTCSE 2016). Chennai, Tamil Nadu, India. Procedia Computer Science, 2016, vol 87, pp. 86-92.

[6] Shailendra Singh et.al. IoT Big data analytics with fog computing for Household energy management in smart grids, Springer nature Second EAI International Conference, SGIoT 2018, Niagara Falls, ON, Canada, July 11, 2018, LNICST, volume 256, pp. 13-22.

[7] V Kamilaris et.al. A review on the practice of Big Data Analysis in Agriculture, Computers and Electronics in Agriculture, 2017, vol 143 (Issue C), pp. 23-37.

[8] Sivaganesan, D, Design and Development Ai-Enabled Edge Computing for Intelligent-IoT Applications, Journal of trends in Computer Science and Smart technology (TCSST),2019, vol 1(issue 02), pp. 84-94.

[9] Rathore et.al. Real-time medical emergency response system: exploiting IoT and big data for public health, 2016, J. Med. Syst.40(12):286

[10] N. Ilyasova et.al. Particular Use of BIG DATA in Medical Diagnostic Tasks, Journal of Science Communication, 2018, vol 28, pp. 114-121.

[11] C.H. Lee et.al. Medical big data: promise and challenges, Kidney Research and Clinical Practice, 2017, vol 36, pp.0311.

[12] Institute for Health Technology Transformation, Transforming Healthcare through Big Data: Strategies for leveraging Big Data in the Healthcare Industry, New York, 2013

[13] F. Andriopoulou et.al. Components and Services for IoT Platforms: Paving the Way for IoT Standards. Springer International Publishing Switzerland, 2016. Integrating IoT and Fog Computing for Healthcare Service Delivery; pp. 213232.

[14] R. S. H. Istepanian, et.al. Internet of M-Health things 'm-IOT', IET Seminar, April 2012, pp.399-405.

[15] R. Singh, A proposal for mobile E-Care health service system using IOT for Indian scenario, Journal of Network Communications and Emerging Technologies, January 2016. vol. 6(1), pp.21-23.

[16] V. Tsoutsouras et.al. Components and Services for IoT Platforms. Springer International Publishing. 2017.15. Software design and optimization of ECG signals analysis and diagnosis for embedded IoT devices.

[17] W. Raghupathi et.al. Big Data analytics in healthcare: Promise and Potential, Health Information Science and Systems, 2014. Vol 2 (3), pp. 01-10.

[18] Hiteshi Pal. Internet of Things (IOT): A Study Analysis of Applications and Benefits in Health Care Sector. IJERT, June 2019.Volume 08, Issue 06. 2278-0181.

[19] B. Xu et.al. The design of an m-Health monitoring system based on a cloud computing plat form. Enterprise Information Systems Talor \& Francis, 2015, Volume 11, Issue 1: The $2^{\text {nd }}$ International Conference on Enterprise Systems. pp. 17-36.

[20] Alex Roehrs et.al. Toward a model for Personal health record Interoperability, IEEE journal of biomedical and health informatics, 2019. vol.23, no 2, pp. 867-873.

[21] C. H. Huang et.al. RFID technology combined with IoT application in medical nursing system, Bulletin of Networking, Computing, Systems, and Software, 2014. vol. 3, no. 1, pp. 20-24.

[22] S. Ge et.al. Design and implementation of interoperable IoT healthcare system based on international standards, in Proc. 9-12 Jan2016, CCNC13. Las Vegas, NV, USA.ISSN: 2331-9860.

[23] G. Muhammad et.al. Smart health solution integrating IoT and cloud: A case study of voice pathology monitoring. IEEE Communications Magazine. January 2017. Volume 55, Issue 1, pp. 69-73.

[24] Bahri Safa et.al. BIG DATA for Healthcare: A Survey, IEEE access, 2019. Vol-7, pp.7397-7408. 
[25] C.H. Lee et.al. Medical big data: promise and challenges, Kidney Research and Clinical Practice, 2017.Vol 36, pp.0311.

[26] Gaspard Harerimana et.al. Health big data analytics: A technology survey, IEEE Access, Nov-2018. Vol- 6, pp. 183186

[27] St. KlimentOhridski et.al. Big data analytics in medicine and Health care. Degruyter/ journal of integrative bioinformatics, May-2018. Volume 15, Issue 3, pp 3-20.

[28] Amir M. Rahmania, et.al. Exploiting smart Ehealth gateways at the edge of the health care Internet of things: A fog computing approach, ELSEVIER/ Future generation computer systems, 2018.Vol-78, part-2, pp. 641-658.

[29] Tahereh Saheb et.al. Paradigm of IoT big data analytics in the healthcare industry: A review of scientific literature and mapping of research trends. ELSEVIER/Telematics and Informatics. 2019. Volume-41, pp. 70-85.

[30] Abdulsalam Yassine et.al. Big data analytics for smart homes with fog and cloud computing, ELSEVIER/ Future generation computer systems, Feb-2019. Vol-91, pp. 563-573.

[31] Mahmood Ahmad et.al. Health Fog: a novel framework for health and wellness applications, Springer/Journal of Supercomputing - Jan-2016, Vol-72, pp. 3677-3695.

[32] Shanhe Yi et.al. Fog Computing: Platform and Applications. IEEE Xplore, Jan-2016, pp.73-78.

[33] Chandy, A. A review on IOT based medical imaging technology for healthcare applications, Journal of Innovative Image Processing (JIIP), 2019. Volume 1(01), pp. 51-60.

[34] Bhatt, C et.al. Internet of things and big data technologies for next-generation healthcare, Studies in Big data. 2017, Volume 30.

[35] TanweerAlam. A Reliable Communication Framework and Its Use in Internet of Things (IoT), International Journal of Scientific Research in Computer Science, Engineering and Information Technology, IJSRCSEIT, 2018, Vol-3, issue 5, pp.450-456.

[36] Iman Azimi1 et.al. Internet of Things for remote elderly monitoring: a study from user-centered perspective. Springer, Journal of Ambient Intelligence and Humanized computing, 2017. Vol-8, pp. 273-289.

[37] Elizalde, D. IoT Hardware - Introduction and Explanation, 2017.

[38] Redowan Mahmud et.al. Cloud-Fog Interoperability in IoT-enabled Healthcare Solutions, ICDCN -18, Jan-2018. Article no-32, pp- 1-10.

[39] G. Matar et.al. Internet of things in sleep monitoring: An application for posture recognition using supervised learning," in Proc. International Conference on IEEE Healthcom, 2016.

[40] Hwang, K et.al. Chen, M. Big-Data Analytics for Cloud, IoT, and Cognitive Computing, 2017, WILEY, ISBN: 978-1$119-24729-6$

[41] Buyya et.al. Internet of Things: Principles and Paradigms. Elsevier, 2016, ISBN: 978-0-12-805395-9.

[42] Gulraiz J. Joyia et.al, Internet of Medical Things (IOMT): Applications, Benefits and Future Challenges in Healthcare Domain, Journal of Communications. April 2017. Vol. 12, No. 4, pp.240-247.

[43] Quwaide et.al. A cloud supported model for efficient community health awareness, Pervasive Mob. Computing. 2016. Volume- 28, pp. 35-50.

[44] Iman Azimi1, Amir M. Rahmani1 et.al. Internet of Things for remote elderly monitoring: a study from user-centred perspective,Springer/Journal of Ambient intelligence and Humanized computing. Apr-2017. Vol-8, pp.273-289.

[45] Min Woo Woo et.al. A reliable IoT system for Personal Healthcare Devices, ELSEVIER, Future Generation Computer Systems, Jan-2018. Vol-78, part2, pp. 173-188.

[46] Gunasekaran Manogaran et.al. A New Architecture of Internet of Things and Big data ecosystem for secured smart healthcare monitoring and alerting, ELSEVIER/Future Generation Computer Systems. May-2018.Vol-82, pp. 375-387.

[47] Kario et.al. Development of a new ICT-based multisensory blood pressure monitoring system for use in hemodynamic biomarker-initiated anticipation medicine for cardiovascular disease: the national IMPACT program project. Prog. Cardiovasc. Dis, Nov-Dec 2017. Volume-60, Issue 3, pp. 435-449. 
[48] Sunder Ali Khowaja et.al. Contextual activity-based Healthcare Internet of things, services and people (HIoTSP): An architectural framework for health monitoring using wearable sensors, ELSEVIER/ computer networks, 2018. Volume 145, pp. 190-206.

[49] Pouria Amirian et.al. Using big data analytics to extract disease surveillance information from point of care diagnostic machines, ELSEVIER/ Mobile and pervasive computing. Dec-2017. Vol -42, pp. 470-486.

[50] Martín-Ruíz et.al. Developing a system for processing health data of children using digitalized toys: ethical and privacy concerns for the Internet of things paradigm. Sci. Eng. Ethics, Springer. 2017, pp. 1-20.

[51] Banos et.al. The mining minds digital health and wellness framework. $3^{\text {rd }}$ International work-conference on Biomatics and Biomed. Eng. (IWBBIO 15), 2016, No. 76.

[52] Kang et.al. "Intelligent personal health devices converged with Internet of things networks". J. Mob. Multimedia, 2017. Volume- 12(3), pp. 197-212.

[53] Vinod Jagannath Kadam, Shivaji rao Manik rao Jadhav, K.Vijayakumar, "Breast Cancer Diagnosis Using Feature Ensemble Learning Based on Stacked Sparse Auto encoders and Soft max Regression”, Image \& Signal Processing, springer, june 2019 .

[54] K. Vijayakumar, K. Pradeep Mohan Kumar, Daniel Jesline, "Implementation of Software Agents and Advanced AoA for Disease Data Analysis", journal of medical systems, Part of Springer Nature 2019. 